\title{
The Unique Acupoint Protocol to Treat Knee Pain of Patient with Thalassemia
}

\author{
Tong Zheng Hong* \\ As-You-Wish Healthcare Institute, Taiwan \\ *Corresponding author: Tong Zheng Hong, As-You-Wish Healthcare Institute, Taiwan
}

\begin{abstract}
This unique and newly developed acupoint protocol composed of Yuan-Source and Luo-Connecting acupoints was conducted to the patient with thalassemia for knee pain. Therapeutic effects of acupuncture for the knee pain were observed and rated by the patient on the Wong-Baker Faces Pain Scale from 6 to 2 with the first treatment, the pain did not reoccur, and the patient could fall asleep easily and soundly, which shows this protocol is effective to reduce the pain and deserve attention.
\end{abstract}

Keywords: Propofol, One-day surgery, Premedication

\section{Introduction}

Those who have thalassemia might experience dizziness, pale skin, shortness of breath, a fast heartbeat, headache, leg cramps, and difficulty concentrating, etc. Thalassemia, an inherited blood disorder that refers to the body makes an abnormal form of hemoglobin, a protein of red blood cells. This disorder can result in excessive destruction of red blood cells carrying to oxygen to all the cells of the body and make the body's red blood cells cannot function properly to finally lead to anemia. The less serious form of this disorder is thalassemia minor. Alpha thalassemia, at least one of the alpha globin genes has a mutation or abnormality and beta thalassemia, the beta globin genes are affected are two main more serious forms $[1,2]$. Up to now, the number of knee pain caused by a sudden injury, an underlying condition, or an overuse injury by has been increasing since it is the most common musculoskeletal complaint. In general, pain is not a symptom associated with thalassemia because it is an unrecognized problem for patients with thalassemia [3]. Ying-Yang is the unique and philosophical concept that serves as the foundation and guideline for the developments of traditional Chinese medicine (TCM) and acupuncture theories like the Five Elements and Qi-Blood. A disease is viewed as the presentation of the imbalance between Ying and Yang. With this concept, the signs and symptoms in the TCM/acupuncture are broader than those in the Western medicine. In general, TCM physicians and acupuncturists usually only depend on the patterns that distinguishes TCM/acupuncture from the Western medicine, instead of following the Western pathological classifications of diseases [4].

\section{Case Presented}

The female patient is 24 suffering from thalassemia and under big stress with the project she oversees. Her chief complaint is left knee pain, which was rated 6 on the Wong-Baker Faces Pain Scale and had been bothering her for more than 2 weeks. Afraid of side effects of medications, she refused to take any pain killers. In addition to distention in the stomach, tinnitus, and bad quality of sleep, she used to wear earplugs during sleep. She also mentioned about the irregular menstrual cycle with only less than 3-day bleeding.

\section{TCM Differential Diagnosis}

Subjective and objective information gathered from the four diagnosis skills, Looking, Listening, Asking, and Pulse Feeling, can be helpful for acupuncture practitioners to consider for synthetic diagnosis and make the effective treatment strategy. 


\section{Looking}

In (Figure 1) the information before treatment from Looking indicated pale complexion, the color of the tongue was slightly pale,

slight white coating on the Lower Jiao, and a little bit of teeth marks on the left side. The tongue was a little bit swollen with a crack at the tip of the tongue.
BEFORE

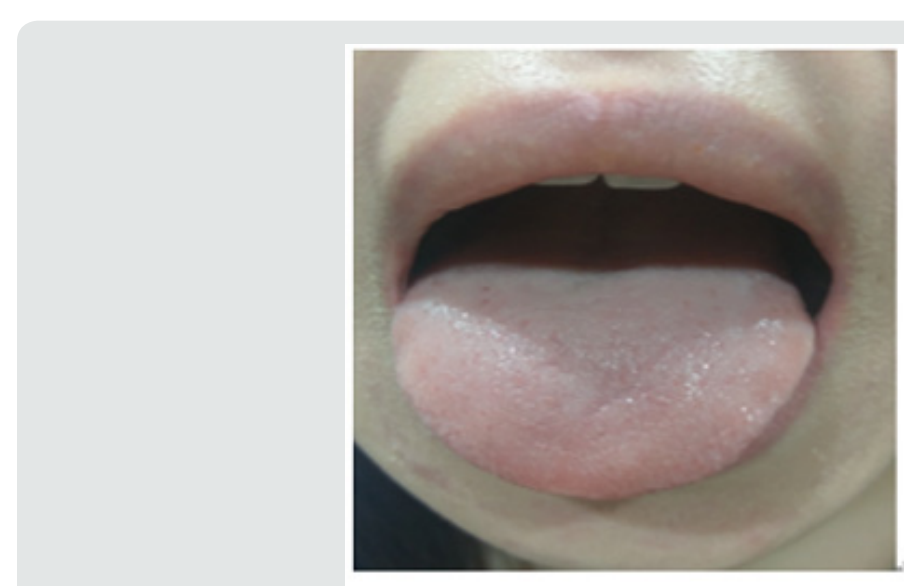

Figure 1: Tongue diagnosis.

\section{Listening}

Her voice was a little bit weak and seemed there was phlegm in the chest when questioned,

\section{Asking}

The patient responded she felt tired easily, especially in the afternoon, hard to fall asleep, and always slept dream disturbed.

\section{Feeling}

Pulse feeling showed wiry, slippery, and thing.

\section{Treatment Plan}

"Less needling for the best outcome" is the golden rule for an acupuncture practitioner to follow since this can enable patients to have a better quality of life in a short time [5]. The unique acupoint protocol Reinforcing Yang to Nourish Yin composed of Yuan-Source acupoints and Luo-Connecting acupoints was considered with the following analysis and consideration of her lifestyle. Most importantly, the manipulation of this protocol takes less than three minutes that can save the time and keep the patient from fear. Thing pulse, low voice, and pale complexion showed Blood deficiency equivalent to anemia in the Western medicine. In addition, the pale and swollen tongue and slight white coating on the Lower Jiao and teeth marks on the left side, a crack at the tip of the tongue are the indications of Blood deficiency. Wiry pulse indicated referred to Liver qi stagnation and phlegm is verified with slippery pulse. The overall information reveals that the pattern of this patient is Blood deficiency, which can also result in insomnia, tinnitus, and difficulty in falling asleep.

\section{Results}

The patient reported the pain was significantly reduced from 6 to 2 on the scale ten minutes right after the first treatment. The color of the tongue turned redder while the white coating vanished a little as shown in (Figure 1). Before the second treatment started the next day, she said she had a sound sleep, tinnitus was relieved, and the knee pain was almost gone. Most importantly, the knee pain did not reoccur with the two treatments. The patient could fall asleep easily and soundly.

\section{Discussion}

Inflammation that can cause pain, redness, swollen joint, joint stiffness, and loss of joint function is a defensive immune response conferred by the host against foreign pathogens [6]. The evidence shows that infected tissues, rheumatic joints, and wounds seem to have lower concentrations of oxygen than healthy tissues [7]. The concept of Ying-Yang in (Figure 2) should deserve attention because it does play a key role to the successful TCM and acupuncture treatments, though is abstract and the unique concept [4]. It is noted in the TCM and acupuncture that Yin referring to materials is the basis of Yang, while Yang is the representations of Zang-Fu organs in exerting Yin [8]. Yin-Yang is incorporated into this acupoint protocol since a disease represents the imbalance between Ying and Yang. This protocol it can enable patients to retrieve the Ying-Yang balance in a shorter time. Acupoints refer to specifically chosen sites that gather Qi in the body and are used to explain the mechanism of acupuncture. Stimulation on different acupoints on the body surface could provide various therapeutic 
benefits. It is presented and discussed in Chapter one of the Volume of the Spirit Pivot in the Huang Di Nei Jing (Yellow Emperor's Inner Classic) that Yuan-Source acupoints are used to treat Zang organs while Fu organs can be treated with Luo-Connecting acupoints in Chapter four. Each of the twelve channels has a Yuan-Source acupoint to pool the Yuan (original) Qi while Luo-Connecting acupoints are connecting Zang and Fu organs bearing the exterior- interior relationship [4]. A human body is constituted by the three essential and vital treasures like Jing (Essence), Qi, and Shen. Jing (Essence) and Blood are viewed as Ying to cooperate with Qi, the energy to benefit the body, which is classified as Yang to maintain functions of the body for transporting Blood to nourish the all the systems of the body to fight against external evil Qi (pathogens).

\section{Ying. \\ Zang \\ Blood \\ Ying channels Material \\ Yang \\ $\mathrm{Fu}$ \\ Qi. \\ Yang channels \\ Function}

Figure 2: Characteristics of Ying and Yang.

The functions of the systems in the body can be affected with the activities of ascent and dispersion of Liver qi. The functions of Liver include storing Blood for regulating the blood volume to nourish the constituents of the body and to regulate the distribution of fluids and blood with free flow of Qi in the traditional twelve channels. The Five Elements theory indicates that the Liver qi can affect the Spleen qi to ascend and the Stomach qi descend correctly, which has a critical impact on the metabolism and oxygen concentration [9].

From the perspectives of the TCM etiology, pain is result of the imbalance of Yin-Yang and that can be classified into 1) deficiency and 2) excess patterns. Both patterns can be resulted from either stagnated Qi or Blood stasis in the systems of the body, especially Liver qi stagnation [4]. The scientific evidence shows the Liver qi stagnation related symptoms can be interpreted with the biological changes of the auto nerve system that can result in issues of the digestion and depression, the improvement of interleukin6,decreasing level of the prostaglandin F $1 \alpha$ (PGF1 $\alpha$ ) levels, increasing level of $\beta$-endorphin and the ratio of 6-keto- PGF1 $\alpha$ / thromboxane B 2 (TXB2) [10]. The countering sequence reveals Liver can invade Spleen to cause deficient Spleen qi, which causes Blood deficiency to transport fluid in the body abnormally and finally cause Dampness. Dampness can cause the presentation of white tongue coating and teeth marks, which means that Spleen Yang is damaged and make stagnated fluids to cause Qi stagnation and Blood stasis [4].

Factors like fear, anxiety, depression, and the lack of sleep can affect how severity of the pain [11].The results of this acupoint protocol complies with the scientific studies revealing that poorer sleep is significantly related to higher levels of nighttime pain [12], which can explain why the patient could sleep soundly with the first treatment. The results suggest this acupoint protocol can reinforce Yang for Zang-Fu organs to nourish Ying to retrieve the balance between Ying and Yang. It is also reported that this protocol has been used either for pain management or other health issues in Table 1 with significant results.

Table 1: Cases of using the protocol.

\begin{tabular}{|c|c|c|}
\hline Diseases & Major Reactions & Pain Reduced/Relieved with Tx \\
\hline Lung Adenocarcinoma & Stage IV, but able to stand and walk with the four treatments. & Four treatments \\
\hline Primary & Menstrual period retrieved within one hour with the first Tx. & - \\
\hline Amenorrhea & Normal blood glucose value with the first Tx & - \\
\hline Diabetes & BP reduced and improved sleep with the first Tx. & One treatment \\
\hline Stomachache & Sleep improved with the first Tx. & Two treatments \\
\hline Disc herniation & Sleep improved with the first Tx. & Two treatments \\
\hline Migraine & &
\end{tabular}




\begin{tabular}{|c|c|c|}
\hline Hypertension & $\begin{array}{c}\text { Chest congestion, headache relieved and sleep improved with } \\
\text { the first Tx. }\end{array}$ & One treatment \\
\hline Osteoporosis & Sleep improved with the first Tx. & One treatment \\
\hline Uterine fibroids & Normal menstrual period the next month. & Two treatments \\
\hline
\end{tabular}

\section{Conclusion}

This unique acupoint protocol benefits the patient effectively to reduce knee pain, enabling her to have a better quality of life in a shorter time. This case suggests referral for acupuncture treatment is a reasonable option for patients with knee pain, particularly for those patients who are afraid of needling. This case shows this protocol can improve the patients' sleep to reduce/relieve pain and help patients stay energetic through activating Ying and Yang at the same time. The reason of successful treatment lies in the accurate identification of Pattern and result of this protocol suggests the balance of Ying-Yang needs more attention in the clinical practice. However, how to understand the mechanism of this protocol with the science-based evidence needs more research in the future since this protocol introduced in this case is totally developed with the limited experiences.

\section{References}

1. Healthline. Everything You Need to Know About Thalassemia.

2. CDC. What is Thalassemia?

3. Greenwalt TJ, Zelenski KR (1984) Transfusion support for haemoglobinopathies. Clin Haematol. 13(1): 151-165.
4. Hong TZ (2019) Managing Cancer Pain with the Exclusive and Newly Developed Reinforcing Yang-Nourishing Yin Acupoint Protocol. Can J Biomed Res \& Tech 1(1): 5 .

5. Hong TZ (2019) Clinical Considerations of Using Extra Acupoints and Traditional Acupoints. Advancements Bioequiv Availab 2(4).

6. Mittal M, Siddiqui MR, Tran K, Reddy SP, Malik AB (2014) Reactive oxygen species in inflammation and tissue injury. Antioxid Redox Signal 20(7): 1126-1167.

7. Nathan C (2003) Oxygen and the inflammatory cell Nature. 2003: 422: 675-676.

8. Hong TZ (2019) Suggestions for Clinical Use of Chinese Herbs. Advancements Bioequiv Availab. 2(3): 153-155.

9. Lu W, Dean-Clower E, Doherty-Gilman A, Rosenthal DS (2008) The value of acupuncture in cancer care. Hematol Oncol Clin North Am. 2008; 22(4): 631-648.

10. Hong TZ (2019) The Evidence-based Views on Liver Qi Stagnation. EC Emergency Medicine and Critical Care 3(2): 96-100.

11. Cancer Research UK. Causes and types of cancer pain.

12. Rumble ME, Keefe FJ, Edinger JD, Affleck G, Marcom PK, et al. (2010) Contribution of cancer symptoms, dysfunctional sleep related thoughts, and sleep inhibitory behaviors to the insomnia process in breast cancer survivors: a daily process analysis. SLEEP 33(11): 1501-1509.
To Submit Your Article Click Here:

This work is licensed under Creative

Commons Attribution 4.0 License

DOI: $10.32474 /$ GJAPM.2020.03.000162

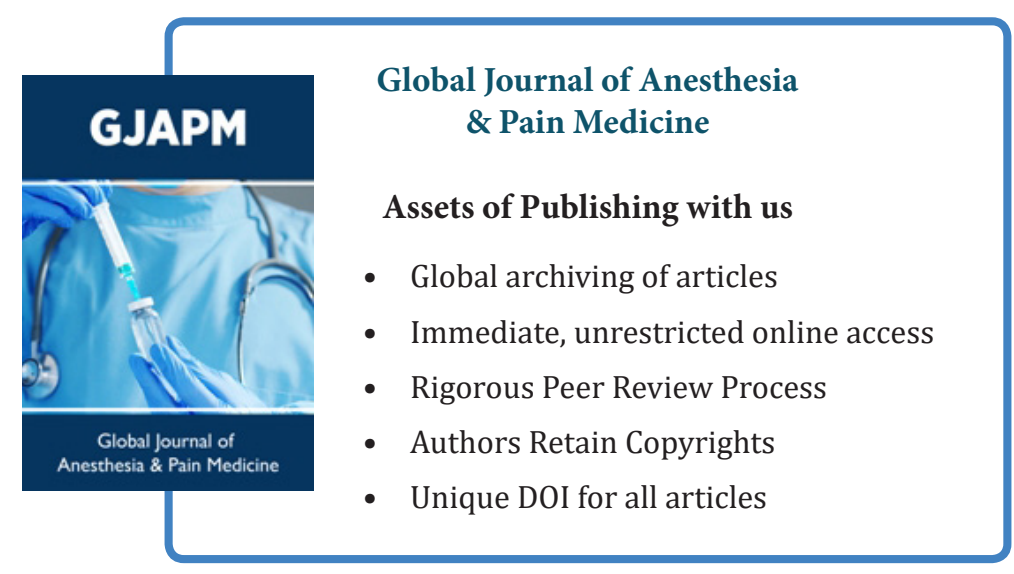

